\title{
INTERAÇÃO QUADRUPOLAR E SUA CORRELAÇĀO COM O EFEITO MÖSSBAUER
}

Sylvestre Schneider

Departamento de Física. Centro de Ciências Naturais e Exatas. UFSM. Santa Maria, RS.

RESUMO

Por meio da expansão em multipolos, chegou-se ao conceito de "momento de multipolo". São dados exemplos de momentos de multi polos. A partir dos mesmos foi definido o "tensor momento de qua drupolo". Partindo da distribuição clāssica de cargas $\rho\left(\vec{r}^{\prime}\right)$ e da ex pansão do potencial em série de Taylor, onde um dos termos da expan são evidencia o momento de quadrupolo, construiu-se a Hamiltoniana clássica em termos de quadrupolo. A expressão quāntica $\hat{H}_{Q}$ para $H_{Q}$ foi obtida, substituindo-se a densidade clássica de carga $\rho(\vec{r})$ pelo operador $\rho^{O P}$, que descreve a situação real em uma distribuição não contínua de cargas.

Us ando a tecnologia dos coeficientes de Clebsh-Gordan e os tensores irredutíveis calculou-se os elementos de matriz de $\hat{H}_{Q}$. Desenvolvida a relação que permite calcular os elementos de matriz da hamiltoniana quântica, aplicou-se a relação obtida a um caso em que se aplica um campo forte sobre um ātomo, obtendo-se uma relação particular para o cálculo dos níveis energéticos da interação qua drupolar.

Fez-se uma aplicação para um átomo ou íon de estado funda mental ${ }^{2} \mathrm{~S}_{1 / 2}$, e spin nuclear $3 / 2$ num campo magnético forte, obten do-se a abertura de níveis para a interação quadrupolar.

A interação quadrupolar foi examinada no efeito Mossbauer para diferentes exemplos.

SUMMARY

SCHNEIDER, S., 1982. Quadrupole interaction and $i$ ts correlaction with the Mossbauer effect. Ciência e Natura(4):1-20.

By means multipole expansion, we guess the concept of "multipole moment". They are given examples of multipole moments. We start of them to define the "quadrupole moment tensor". By starting from the classical chargedensity distribution $\rho\left(\vec{r}^{\prime}\right)$ and from the potential expanded in a Taylor series, in which one one of the terms allows us to see the quadrupole moment, we construct a classical Hamiltonian in terms of quadrupole. The quantum-mechanical expression $\bar{H}_{Q}$ por $H_{Q}$ is given, by substitution of the classical charge density $\rho(\vec{r})$ by an operator $\rho(O p)$, which describes adequately the real con figuration in a non-continuous charge distribution. By use of the 
Clebsh-Gordan tecnology-coefficients with the irredutible tensors, the matrix-elements of $\hat{H}_{Q}$ was calculated. The relation develloped, which allows the calcule of the matrix-elements of $\bar{H}_{Q}$, this is used for an application to a specific case, of a strong magnetic field excerted on an atom. It is obtained a particular relation, for cal culating the energy levels of quadrupolar interaction. The sequent purpose was to work out an application for an atom or ion in the fundamental state ${ }^{2} \mathrm{~S}_{1 / 2}$, and nuclear spin $3 / 2$ in a strong magnetic field. The result was a splitting in energy levels for the quadrupo lar interaction.

The quadrupolar interaction was examined in correlation of the Mossbauer-Effect in different examples.

\section{INTRODUÇAO}

Ao estudar-se os fenômenos nucleares não se podem conside rar somente as interações magnēticas do nūcleo com suas vizinhanças. Considerando o efeito sobre a carga nuclear esta determina as órbi tas eletrônicas e onde o nūcleo está situado na mólécula. Deve-se tambēm levar em conta alguns efeitos elétricos sobre a energia ne cessāria para reorientar o núcleo. Que estes efeitos existem pode ser visto ao considerar-se um nūcleo não esférico. Suponhamos que ele seja algo alongado e que seja atuado pelas cargas mostradas na Figura 1 .

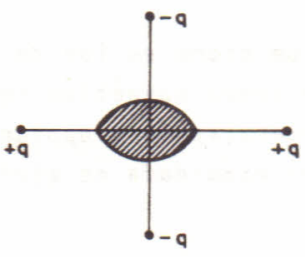

(a)

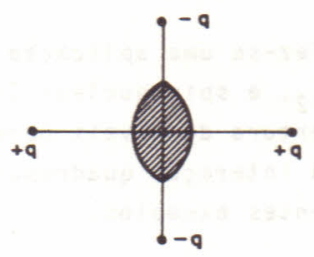

(b)

Figura 1. (a) Um nūcleo de forma alongada no campo de quatro cargas, $+q$ sobre o eixo $x$; $-q$ sobre o eixo y. A configuração de (b) é energeticamente mais favorāvel porque põe as cargas positivas nas extremidades da forma alongada mais próximas das cargas negativas.

A Figura 1(b) corresponde a uma energia mais baixa, por que tem colocado os tipos de carga nuclear positiva mais próximas às cargas externas negativas. Hā, portanto uma energia eletrostátị ca que varia com a orientação nuclear. Naturalmente, girando o nú cleo de extremidade à extremidade, não afeta a energia eletrostātí ca. Conseqüentemente, para núcleos de spin $1 / 2$ a energia eletrostātí 
ca não abre a degenerāncia $\mathrm{m}_{\mathrm{I}}$.

Aqui desenvolvemos uma teoria mais quantitativa, por meio de uma descrição em termos da densidade de carga clāssica, do nū cleo, $\rho$, . Obteremos uma resposta quântica substituindo o $\rho$ clāssico pelo seu operador quântico.

Consequentemente, construímos o Hamiltoniano de quadrup o 10 que calculamos. Os resultados quantitativos serão relacionados ao efeito Mossbauer, por meio de exemplos apresentados.

\section{DESEN VOLVIMENTO}

Expansão em Multipolos

Consideremos uma distribuição de cargas caracterizada por $\rho\left(\vec{r}^{\prime}\right)$; por hipótese, $\rho\left(\vec{r}^{\prime}\right) \neq 0$ apenas no interior de uma esfera de raio $R$, centrada em uma origem conveniente.

Uma das maneiras possiveis de se escrever o potencial de vido a $\rho\left(\vec{r}^{\prime}\right)$ é em termos de harmônicos esféricos, conforme Jackson (2):

$$
\Phi(\vec{r})=\sum_{\ell=0}^{\infty} \sum_{m=-\ell}^{\ell} \frac{4 \pi}{2 \ell+1} q_{\ell m} \underline{y_{\ell m}(\theta, \phi)}
$$

A expressão [1] é chamada EXPANSAO EM MULTIPOLOS; para $\ell=0$, temos o monopolo; para $\ell=1$, temos o dipolo, etc...

Nosso interesse aqui é a determinação das constantes $q_{\ell m}$. Para isto, podemos usar outra expressão para o potencial:

$$
\Phi(\vec{r})=\int \frac{\rho\left(\vec{r}^{\prime}\right)}{\left|\vec{r}-\vec{r}^{\prime}\right|} d v^{\prime}
$$
se usarmos agora a expansão de $\frac{1}{\left|\vec{r}-\vec{r}^{\prime}\right|}$ em harmônicos esféricos, a ob
tenção dos $q_{\ell m}$ será imediata:

$$
\frac{1}{\mid \overrightarrow{r-r^{\prime} \mid}} 4 \pi \sum_{\ell=0}^{\infty} \sum_{m=-\ell}^{\ell} \frac{1}{2 \ell+1} \frac{r<\ell}{r>\ell+1} y^{*}{ }_{\ell m}\left(\theta^{\prime}, \phi^{\prime}\right) y_{\ell m}(\theta, \phi), \quad[3]
$$

(2)

Nesta expressão consideraremos $r<=r^{\prime}$ e $r>=r$, levando em

$$
\begin{array}{r}
\Phi(\vec{r})=4 \Pi \sum_{\ell=0}^{\infty} \sum_{m=-\ell}^{\ell} \frac{1}{2 \ell+1} \frac{1}{r^{\ell+1}} y_{\ell m}(\theta, \phi) \int \rho(\vec{r}) r^{\prime l} y_{\ell, m}^{*}\left(\theta^{\prime}, \phi^{\prime}\right) d v^{\prime}[4] \\
\text { Comparando [4] e [1]: } q_{\ell m}=\int \rho(\vec{r}) r^{\prime l} y_{\ell, m}^{*}\left(\theta^{\prime}, \phi^{\prime}\right) d v^{\prime} \quad[5]
\end{array}
$$

os que são chamados "MOMENTO DE MULTIPOLO".

Exemplos de cáleulo de $q_{\mathrm{lm}}$

Para calcularmos os $q_{\ell m}$, necessitamos evidentemente, do 
conhecimento dos $y_{\ell m}$, alguns dos quais estão listados a seguir:

$$
\begin{aligned}
& \ell=0 \quad Y_{00}=\frac{1}{\sqrt{4 \Pi}} \quad \ell=1 \quad Y_{11}=-\sqrt{\frac{3}{8 \pi}} \operatorname{sen} \theta \ell^{i \phi} ; Y_{10}=\sqrt{\frac{3}{4 \pi}} \cos \theta \\
& \ell=2 \quad Y_{22}=\frac{1}{4 \pi} \sqrt{\frac{15}{4 \pi}} \operatorname{sen}^{2} \theta \ell^{2 i \phi} ; y_{21}=-\sqrt{\frac{15}{8 \Pi}} \operatorname{sen} \theta \cos \phi \ell^{i \phi} ; \\
& Y_{20}=\sqrt{\frac{5}{4 \pi}}\left(\frac{3}{2} \cos ^{2} \theta-\frac{1}{2}\right. \\
& \text { Teremos então }
\end{aligned}
$$$$
q_{10}=\int \rho(\vec{r}) r^{\prime}\left(\frac{3}{4 \pi}\right)^{1 / 2} \cos \theta d v^{\prime}=\left(\frac{3}{4 \pi}\right)^{1 / 2} \int z^{\prime} \rho\left(\vec{r}^{\prime}\right) d v^{\prime}=\left(\frac{3}{4 \pi}\right)^{1 / 2} \rho z
$$

Aqui usamos a definição de momento de dipolo elétrico:

$\vec{\rho}=\int \vec{r}^{\prime} \rho\left(\vec{r}^{\prime}\right) \mathrm{d} v^{\prime}$

$q_{11}=\int \rho\left(\vec{r}^{\prime}\right) r^{\prime}\left[-\left(\frac{2}{8 \pi}\right)^{1 / 2}\right] \operatorname{sen} \theta e^{i \phi} d v^{\prime}$

Mas $r^{\prime} \operatorname{sen} \theta e^{i \phi}=r^{\prime} \operatorname{sen} \theta \cos \phi+i r^{\prime} \operatorname{sen} \theta \operatorname{sen} \phi=x^{\prime}-i y^{\prime}$

.

$$
\begin{aligned}
& q_{11}=-\left[\frac{3}{8 \pi}\right]^{1 / 2} s \rho\left(\vec{r}^{\prime}\right)\left(x^{\prime}-i y^{\prime}\right) d v^{\prime}=-\left[\frac{3}{8 \pi}\right]^{1 / 2}\left(\rho x^{\prime}-i \rho y^{\prime}\right) \\
& q_{22}=\frac{1}{4 \pi}\left[\frac{15}{2 \pi}\right] \quad \rho \rho\left(\vec{r}^{\prime}\right) r^{2} \operatorname{sen}^{2} \theta e^{2 i \phi} d v^{\prime}=\frac{1}{4 \pi}\left[\frac{15}{2 \pi}\right]^{1 / 2} \rho \rho\left(\vec{r}^{\prime}\right) \\
& {\left[x^{\prime}-i y^{\prime}\right]^{2} d v^{\prime}}
\end{aligned}
$$

$$
\text { Porque } r^{\prime 2} \operatorname{sen}^{2} \theta l^{2 i \phi}=\left(r^{\prime} \operatorname{sen} \theta l^{i \phi}\right)^{2}=\left(x^{\prime}-i y^{\prime}\right)^{2}=
$$

$=x^{\prime 2}-2 i x^{\prime} y^{\prime}-y^{\prime 2}$

E conveniente agora definirmos o tensor momento de quadru polo: Teremos:

$$
\begin{aligned}
& Q_{11}=\int\left(3 x^{\prime 2}-r^{\prime 2}\right) \rho\left(r^{\prime}\right) d v^{\prime} ; Q_{12}=\int 3 x^{\prime} y^{\prime} \rho\left(r^{4}\right) d v^{\prime}=Q_{21} \\
& Q_{22}=\int\left(3 x^{\prime 2}-r^{\prime 2}\right) \rho\left(r^{\prime}\right) d v^{\prime} ; \text { etc... }
\end{aligned}
$$

Então:

$$
q_{22}=\frac{1}{12} \frac{15}{2 \pi}^{1 / 2} s\left(3 x^{\prime 2}-6 i x^{\prime} y^{\prime 2}\right) \rho\left(r^{\prime}\right) d v^{\prime}=\frac{1}{12}_{2 \pi}^{15 / 2} Q_{11^{-2 i Q_{12}}}{ }^{-Q_{22}}
$$

Analogamente, obteremos: 


$$
q_{21}=-\frac{1}{3} \frac{15}{8 \pi}^{1 / 2}\left(Q_{13}-i Q_{23}\right) ; q_{20}=\frac{1}{2} \frac{5}{4 \pi}^{12} Q_{33}
$$

Observação: sendo $Y_{\ell,-m}(\theta, \phi)=(-1)^{m} Y_{\ell,-m}^{*}(\theta, \phi)$, teremos:

$$
q_{\ell, m}=(-1)^{m} q_{\ell, m} \text {, desde que a densidade de cargas seja real. }
$$

\section{HAMILTONIANA CLASSICA}

Suponhamos que a distribuição de cargas $\rho\left(\vec{r}^{\prime}\right)$ esteja suje ta a um potencial devido a fontes externas. A energia de interação entre este potencial e $\rho\left(\vec{r}^{\prime}\right)$ :

$$
W=\int \rho\left(\vec{r}^{\prime}\right) V\left(\vec{r}^{\prime}\right) d v^{\prime}
$$

$V\left(\vec{r}^{\prime}\right)$ pode ser expandido em sērie de Taylor em torno de uma origem conveniente:

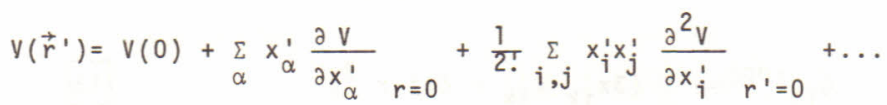

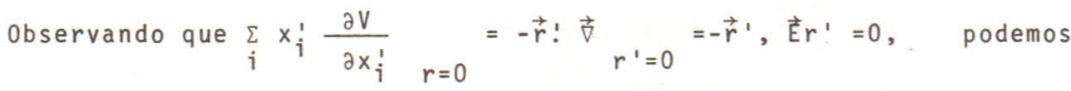
reescrever os dois ūitimos termos de [9]:

$$
V(\vec{r})=V(0)-\vec{r}^{\prime}, \vec{E}-\frac{1}{2 !} \sum_{i, j} x_{i}^{\prime} x_{j}^{\prime} \frac{\partial E_{i}}{\partial x_{i}^{\prime}}+\ldots
$$

Na região livre de cargas, $\vec{\nabla}, \vec{E}=0$. Em particular para o nos so campo externo, na região de nosso interesse, isto ē sempre verda de. Podemos então, subtrair do ūitimo termo de [10] a expressão nú $1 \mathrm{a}$

$$
\frac{1}{6} r^{2} \vec{\nabla} \cdot \vec{E}(0):
$$

$$
V\left(\vec{r}^{\prime}\right)=V(0)-\vec{r}^{\prime} \cdot \vec{E}(0)-\frac{1}{6} \sum_{i, j}\left(3 x_{i}^{\prime} x_{j}^{\prime}-r^{\prime 2} \delta i j\right) \frac{\partial E_{j}}{\partial x_{i}}(0)+\ldots[11]
$$

Levando 11 em 8 , virā:

$$
W=V(0) q-\vec{\rho} \cdot \vec{E}(0)-\frac{1}{6} \sum_{i, j} Q_{i j} \frac{\partial E_{j}}{\partial x_{j}}(0)+\ldots
$$

Estas forma de escrever a energia tem a vantagem de mos trar as diversas interações: carga e potencial, dipolo-campo, qua drupolo, gradiente do campo, etc.

De [12], podemos tirar imediatamente a Hamiltoniana corres pondente ao termo de quadrupolo: 


$$
H_{Q}=\frac{1}{6} \underset{i, j}{\Sigma} Q_{i j} \frac{\partial E_{j}}{\partial x_{i}^{T}}
$$

\section{EXPRESSÃO QUÂNTICA PARA O $\mathrm{H}_{\mathrm{Q}}$}

Para obtermos o operador $\hat{H}_{R}$, devemos substituir a densida de clássica, $\rho(\vec{r})$, pelo operador $\rho(o p)^{\rho}$, que descreve a situação real em uma distribuição não contínua de cargas. E fácil compreender que tal situação é descrita por:

$$
\begin{gathered}
\rho^{(o p)}=e \sum_{k=1}^{z} \delta\left(\vec{r}, \vec{k}_{k}\right) \text {, para um núcleo atômico, }[14] \text { onde temos: } \\
\text { e=carga dos prótons; } k=i \text { ndice para os prótons }(k=1,2 \ldots z) . \\
\text { Levando }[14] \text { na definição de } Q_{i j} \text {, virā } \\
Q_{i j}(o p)=\delta 3 x_{i}^{\prime} x_{j}^{\prime}-\delta i j r^{\prime 2} \sum_{k=1}^{z} \text { e } \delta\left(\vec{r}^{\prime}-\vec{\rho}_{k}^{\prime}\right) d v^{\prime}
\end{gathered}
$$$$
\cdot
$$$$
Q_{i j}(o p)=e \sum_{k}\left(3 x_{i k}^{\prime} x_{j k}^{\prime}-\delta i j r^{\prime 2}\right)
$$

Com [15], podemos escrever:

$\hat{H}_{Q}=\frac{1}{6} \sum_{i, j} Q_{i j}$ (op) $\frac{\partial E_{j}}{\partial x_{i}^{\prime}}$

Nosso próximo passo será o cālculo dos elementos de mạ triz de $\hat{H}_{Q}$. A representação que usaremos será caracterizada pelos seguintes números quânticos:

1. Momento angular total do núcleo: I

2. As $(2 I+1)$ componentes de $I: m$

3. Conjunto de todos os outros números quânticos que não interessa especificar aqui: $\tau$

Como estamos interessados apenas na reorientação espacial do núcleo para um dado estado de energia, necessitamos apenas elemen tos diagonais em $I$ e $\tau$. Assim, os elementos procurados serão da for ma :

$$
<\tau \operatorname{Im}\left|Q_{i j}(O p)\right| \tau^{\prime} I m>
$$

Entretanto, para o cálculo destes elementos, vamos usaros coeficientes de Clebsh-Gordan, o conceito de tensores irredutíveis e o teorema de Wigner'Eckart, sobre os quais falaremos na secção se guinte.

COEFICIENTES DE CLEBSH-GORDAN: CONCEITO

os coeficientes de Clebsh-Gordan (C.G.) estão intimamente 
relacionados com a soma de momentos angulares, em mecânica quântica (3).

Considere um sistema quāntico constituido de duas partes: uma caracterizada pelos nümeros quânticos $L$, $M$ e por $\psi_{L, M}$; o outro por $J^{\prime}, M_{J}{ }^{\prime}$ e $\psi_{J}{ }^{\prime}, M_{j}$. O O sistema todo será caracterizado por $J, M$ e $\psi_{J M_{J}}$. Então, a função $\psi_{J M_{J}}$ pode ser expressa como uma combinação linear de produtos de funções das duas partes do sistema (se estes produtos formam um conjunto completo):

$$
\psi_{J M_{J}}=\sum_{J, M_{J},} C\left(J^{\prime}, L, J ; M_{J}, M, M_{J}\right) \psi_{J} M_{J}, \psi_{L M} \quad[17]
$$

os $C\left(J^{\prime}, L, J ; M_{J}, M, M_{J}\right)$ são os coeficientes de ClebshGordan, sua determinação estā indicada nos livros de Teoria de Gru po e na Mecânica Quântica.

Tensores irredutiveis: conceito e exemplo

Existem diversas maneiras de conceituar tensor irredutí vel, entre elas, a que se segue nos parece a mais apropriada para os objetivos do trabalho.

Seja um sistema quântico cujo momento angular $\tilde{\vec{j}}$ tenha com ponentes $\hat{j}_{n}, \hat{j}_{y}$ e $\hat{j}_{z}$

Sabemos que, a partir destes operadores, podemos definir:

$$
J+=J_{n}+i J_{y} \text { e } J-=J_{n}-i J_{y}
$$

Podemos tambēm construir funções $G$ destes operadores dos sistemas e examinar comutadores do tipo J+,G, J-,G, J,$G$ etc... Podemos tambëm construir uma famīlia de $2 \ell+1$ operadores in diciados por $M(M=L, L-1, \ldots,-L)$. Esta familia, denotada por $T_{L M}$, será chamada "de operadores tensoriais irredutíveis" se as seguintes relações de comutação forem satisfeitas:

$$
\begin{aligned}
& J_{ \pm}, T_{L M}=L(L+1)-M(M+1)^{1 / 2} T_{L M+} \\
& J_{Z}, T_{L M}=M T_{L M}
\end{aligned}
$$

Exemp10: De $[20]: J_{z}, T_{L M}=J_{z}, T_{10}=0$. Isto nos mostra que po demos construir uma famīlia de tensores $T_{L M}$ partindo de um $T_{10}$ que comute com J $\mathrm{J}_{z}$. Podemos tentar,:por exemplo, $\mathrm{T}_{10}=\mathrm{J}_{z}$ que, evidentemen te comuta, consigo mesmo.

$$
\operatorname{Se}(\overline{I V} .3): J+, T_{10}=\sqrt{2} T_{11}=J+, J_{Z} \cdot \because T_{11}=-J+/ \sqrt{2}
$$


pois $J+, J z=-J+$

$$
\text { e } \mathrm{J}-, \mathrm{T}_{10}=\sqrt{ } 2 \mathrm{~T}_{1-1}=\mathrm{J}-, \mathrm{J}_{2}=\mathrm{J}-\therefore \mathrm{T}_{1-1}=\mathrm{J}-/ \sqrt{ } 2
$$

Teorema de Wigner-Eckart

Considere um conjunto de funções de onda $\left|\tau J M_{J}\right\rangle,\left|\tau{ }^{\prime} J^{\prime} M_{J}{ }^{\prime}\right\rangle$ etc... Estamos interessados em calcular os elementos de matriz de operadores $T_{\text {LM }}$, usando tais vetores como base. 0 teorema de Wigner Eckart (1) estabelece que tais elementos de matriz estão relaciona dos com os coeficientes de Clebsh-Gordan atravēs de conjunto de quan tidades $<\tau J|| T_{L}|| \tau J^{\prime}>$, que não dependem de $M, M_{J}, M_{J}$, ou:

$\left\langle\tau J_{M J}\left|T_{L M}\right| \tau^{\prime} J^{\prime} M_{J}{ }^{\prime}\right\rangle=C\left(J^{\prime}, L, J ; M_{J}, M, M_{J}\right)\left\langle\tau J|| T_{L}|| \tau^{\prime} J^{\prime}\right\rangle$

Regras de selegão

Sabemos que, em certos casos, é possĩvel deduzir elemen tos de matriz de operadores utilizando regras de comutação envolven do estes operadores. Em particular, isto é sempre possível quando os operadores estão relacionados com momento angular. E sabido que quando um operador comuta com H (operador hamiltoniano), as autofun ções são comuns.

Consideremos um conjunto de operadores que comutam: $\mathrm{J}^{2}, \mathrm{~J}_{2}$ e outros operadores com autovalores $\mathrm{J}, \mathrm{M}_{\mathrm{J}}$ e $\tau$.

Estamos interessados em elementos de matriz do tipo:

$$
<\tau J M_{J}\left|T_{L M}\right| \tau ' J M_{J}>
$$

De 20 deduzimos que:

$$
\left\langle\tau J M_{J}\left|J_{Z}, T_{L M}\right| \tau{ }^{\prime} J^{\prime} M_{J},\right\rangle=M\left\langle\tau J M_{J}\left|T_{L M}\right| \tau{ }^{\prime} J^{\prime} M_{J}{ }^{\prime}\right\rangle
$$

Mas :

$$
\left\langle\tau J M_{J}\left|\left(J_{Z} T_{L M}-T_{L M} J_{z}\right)\right| \tau^{\prime} J^{\prime} M_{J^{\prime}}>=\left(M_{J}-M_{J^{\prime}}\right)<\tau J M_{J}\left|T_{L M}\right| \tau^{\prime} J^{\prime} M_{J^{\prime}}>\right.
$$

Então:

$$
\begin{aligned}
& \left(M_{J}-M_{J^{\prime}}\right)<\tau J M_{J}\left|T_{L M}\right| \tau{ }^{\prime} J^{\prime} M_{J}{ }^{\prime}>=M<\tau J M_{J}\left|T_{L M}\right| \tau^{\prime} J^{\prime} M_{J^{\prime}}> \\
& \text { - } \\
& \left\langle\tau J M_{J}\left|T_{L M}\right| \tau^{\prime} J^{\prime} M_{J^{\prime}}>=0 \text { a não ser que } M_{J}-M_{J^{\prime}}=M\right.
\end{aligned}
$$

Antes de trabalharmos esta expressão, observemos que:

$J_{-}|j m\rangle=k|j m-1\rangle$. Tomando o conjunto hermitiano desta expres são, $v i r a ̄<j m \mid J_{+}=\langle j m-1| K \quad$ ( $k$ : constante, real). 
Então:

$$
\begin{aligned}
& \left.<\tau J M_{J}\left|J_{ \pm}, T_{L M}\right| \tau{ }^{\prime} J M_{J}\right\rangle=J(J+1)-M_{J}\left(M_{J} \mp 1\right)^{1 / 2}\left\langle J M_{J} \mp\right. \\
& \mp 1 . \tau\left|T_{L M}\right| \tau{ }^{\prime} J M_{J} M^{\prime}>
\end{aligned}
$$

e

$$
\begin{aligned}
& <\tau J M_{J}\left|T_{L M} J \pm\right| \tau^{\prime} J^{\prime} M_{J}>=J^{\prime}\left(J^{\prime}+1\right)-M_{J}\left(M_{J}{ }^{\prime} \pm 1\right) 1 / 2< \\
& \left\langle\tau J M_{J}\left|T_{L M}\right| \tau^{\prime} J^{\prime} M_{J}{ }^{\prime} \pm 1>\right.
\end{aligned}
$$

Levando [24] e [25] em 23 , virā:

$$
\begin{aligned}
& \left.J(J+1)-M_{J} \mp 1\right)^{1 / 2}<\tau J M_{J} \mp 1\left|T_{L M}\right| \tau^{\prime} J^{\prime} M_{J}{ }^{\prime}>J^{\prime}\left(J^{\prime}+1\right)- \\
& -M_{J}\left(M_{J}{ }^{\prime} \pm 1\right) 1 / 2 .<\tau M_{J}\left|T_{L M}\right| J^{\prime} M_{J} J^{\prime} \pm \tau^{\prime}>=L(L+1)- \\
& -M(M \pm 1) \quad 1 / 2<\tau J M_{J}\left|T_{L M} \pm 1\right| \tau^{\prime} J^{\prime} M_{J} J^{\prime}>
\end{aligned}
$$

Os únicos termos desta equação que não desaparecem são os que satisfazem a equação [22]. $M_{J}-M_{J}=M$. As equações [22] e [26], constituem um conjunto de relações de recorrência entre os TLM e en tre os $T_{L M}$ e $T_{L M+1}$. Elas são suficientes para permitir o cálculodos elementos de matriz para dados J, J', $\tau, \tau^{\prime}$ em termos de um outro elemento qualquer.

Vamos necessitar ainda de deduzir uma outra relação, entre elementos de matriz de $T_{L M}$, onde os $T_{L M}$ são funções de variāveis dị ferentes.

$$
\begin{array}{r}
\text { Para tanto, consideremos o operador } J_{z}=L_{z}+J_{z} \text {, onde } \\
J_{Z}\left|J_{M-}>=M_{J}\right| J M_{J}>; L_{z}|L M>=M| L M>; J_{z}^{\prime}\left|J M_{J}>=M_{J}\right| J^{\prime} M_{J}>
\end{array}
$$

Então

$$
<L M^{\prime} M_{J},\left|J_{Z}\right| J M_{J}>=M_{J}<L M^{\prime} \mid J M_{J}>=M_{J} C\left(L M J^{\prime} M_{J} \cdot \mid J M_{J}\right)
$$

Aqui, $J_{z}$ atuou à direita. Escrevendo $J_{z}=L_{z}+J_{z}^{\prime}$ e fazendo-o atuar à esquerda:

$$
\left.<L M J^{\prime} M_{J} \cdot\left|L_{Z}+J_{Z}^{\prime}\right| J M_{J}\right\rangle=\left(M+M_{J}\right) C\left(L M J^{\prime} M_{J} ; j J M_{J}\right)
$$

e virā

$$
\left(M_{J}+M_{J}\right) C\left(L M J M_{J} ; J M_{J}\right)=M C\left(L M J M_{J} ; J M_{J}\right)
$$

que é uma relação de recorrência para os coeficientes de clebsh-Gor dan idêntica à relação [22] para os $T_{L M}$. Pode-se mostrar também que os coeficientes de C.G. obedecem a uma relação idêntica a [26]. En tão, podemos afirmar que os elementos de matriz de $T_{L M}$ e os coeficien tes de C.G. estão relacionados. Esta relação é o teorema de Wigner Eckart [5]. 
No nosso exemplo de tensores irredutiveis, vimos que, pa ra dados L e M, podemos construir vārios $T_{L M}$, pois $1 \bar{a}$, escolhemos um deles arbitrariamente. Mas, os coeficientes de C.G. São os mesmos pa ra tensores diferentes de mesmo $L$ e $M$. Apenas a constante $\left\langle\tau\left\|T_{L}\right\| \tau^{\prime} J^{\prime}\right\rangle$ dependerā das variāveis usadas.

Para ilustrar este ponto, consideremos uma partícula de spin $\vec{S}$, momento angular orbital $\vec{\ell}$ e posição $\vec{r}$. 0 momento angular to tal $\bar{e}$ dado por $\vec{\jmath}=\vec{\imath}+\vec{S}$.

Podemos então, usando [19] e [20] que são regras de comu tação, construir os $T_{L M}$ para $L=2$, como função de $\vec{j}$ ou de $\vec{r}$. Em vista da relação $\mathrm{J}_{z}, \mathrm{~T}_{22}=2 \mathrm{~T}_{22}$, devemos escolher para $\mathrm{T}_{22}$ uma função que, ao comutar com $\mathrm{J}_{z}$, dá ela mesma em dobre. Sabemos que funções de $\mathrm{J}^{+}$ podem vir a satisfazer tal relação. Em particular $j_{+}^{2}$ satisfaz. Tere mos pois, $\mathrm{T}_{22}=\mathrm{J}_{+}^{2}$

$$
\text { De } 19 \mathrm{~J}-, \mathrm{J}_{+}^{2}=6-2^{1 / 2} \mathrm{~T}_{21}=2 \mathrm{~T}_{21} \quad \mathrm{~J}+, \mathrm{J}_{\mathrm{z}}=-\mathrm{J}+
$$

e

$$
\mathrm{J}-, \mathrm{J}^{2}+=\mathrm{J}-\mathrm{J}+\mathrm{J}+-\mathrm{J}+\mathrm{J}+\mathrm{J}-; \text { Mas } \mathrm{J}-, \mathrm{J}+=-2 \mathrm{z}
$$

\section{Então}

$$
\begin{aligned}
& \mathrm{J}-, \mathrm{J}+=\mathrm{J}-\mathrm{J}+\mathrm{J}+-\mathrm{J}+\mathrm{J}+\mathrm{J}-=(-5 \mathrm{z}+\mathrm{J}+\mathrm{J}-) \mathrm{J}+-\mathrm{J}+(\mathrm{J}-\mathrm{J}+-5 \mathrm{~g})= \\
& =-2\left(\mathrm{~J}_{\mathrm{z}} \mathrm{J}+-\mathrm{J}+\mathrm{J}_{\mathrm{z}}\right) \cdot \therefore \mathrm{T}_{21}=-\left(\mathrm{J}_{\mathrm{z}} \mathrm{J}++\mathrm{J}+\mathrm{J}_{\mathrm{z}}\right) \\
& \text { De } 19 \mathrm{~J}-, \mathrm{T}_{21}=\sqrt{ } 6 \mathrm{~T}_{20} ; \mathrm{J}-,-\left(\mathrm{J}_{\mathrm{Z}} \mathrm{J}++\mathrm{J}+\mathrm{J}_{\mathrm{Z}}\right)=-
\end{aligned}
$$

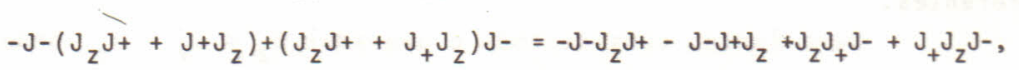

$$
\begin{aligned}
& \text { onde } \mathrm{J}+\mathrm{J}_{\mathrm{z}}=\mathrm{J}+, \mathrm{J}_{\mathrm{z}}=\mathrm{J}_{\mathrm{z}}, \mathrm{J}+ \\
& =-\mathrm{J}-\mathrm{J}_{\mathrm{z}} \mathrm{J}+-\mathrm{J}-\mathrm{J}_{\mathrm{z}} \mathrm{J}+-\mathrm{J}++\mathrm{J}_{\mathrm{z}} \mathrm{J}+\mathrm{J}-\mathrm{J}_{\mathbf{z}} \mathrm{J}+-\mathrm{J}+\mathrm{J}-= \\
& =2 \mathrm{~J} z+\mathrm{J}-2 \mathrm{~J}-\mathrm{J} z \mathrm{~J}+\mathrm{J}-\mathrm{J}+-\mathrm{J}+\mathrm{j}- \\
& \text { Mas } \mathrm{J}_{\mathrm{z}} \mathrm{J}+\mathrm{J}-=\mathrm{J}_{\mathrm{z}} \mathrm{J}-\mathrm{J}+2 \mathrm{~J}_{\mathrm{z}}=\mathrm{J}_{\mathrm{z}} \mathrm{J}-\mathrm{J}+2 \mathrm{~J}_{\mathrm{z}}^{2}= \\
& =\mathrm{J}-\mathrm{J} z-\mathrm{J}-\mathrm{J}++2 \mathrm{~J}_{\mathrm{z}}^{2}=\mathrm{J}-\mathrm{J} z+-\mathrm{J}-\mathrm{J}+2 \mathrm{~J}_{\mathrm{z}}^{2} \\
& \therefore \quad \mathrm{J}-\mathrm{T}_{21}=2 \mathrm{~J}-\mathrm{J} z \mathrm{~J}+-2 \mathrm{~J}-\mathrm{J}+\mathrm{J} 4 \mathrm{~J}_{\mathrm{z}}^{2}-2 \mathrm{~J}-\mathrm{J}_{\mathrm{z}} \mathrm{J}+-2 \mathrm{~J} z= \\
& =-2 J^{2}-J_{z}^{2}-J_{z}+4 J_{z}^{2}-2 J_{z}=6 J_{z}^{2}-2 J^{2}
\end{aligned}
$$

Então $T_{20}=\frac{1}{\sqrt{ } 6} 23 \mathrm{~J}_{\mathrm{z}}^{2}-\mathrm{J}^{2}=\sqrt{ } \frac{2}{3} 3 \mathrm{~J}_{\mathrm{z}}^{2}-\mathrm{J}^{2}$

$$
\text { De } 19 \mathrm{~J}-, \mathrm{T}_{20}=6^{1 / 2} \mathrm{~T}_{2,-1}
$$$$
J-,(2 / 3)^{1 / 2}\left(3 J_{z}^{2}-J^{2}\right)=(2 / 3)^{1 / 2} J-\left(3 J_{z}^{2}-J^{2}\right)-\left(3 J_{z}^{2}-J^{2}\right) J-=
$$ 


$$
\begin{aligned}
& =(2 / 3)^{1 / 2} 3 J-J_{z}^{2}-J-J^{2}-3 J_{z}^{2} J-+J^{2} J-=\sqrt{6} J-J_{z} J-J z J j J-= \\
& =\sqrt{6}\left(J-J_{z}+J-\right) J_{z}-J_{z}\left(J-J_{z}-J-\right)=\sqrt{6} J-J_{z}+J_{z} J- \\
& \therefore \mathrm{T}^{2-1}=\mathrm{J}-\mathrm{J}_{2}+\mathrm{J}_{z} \mathrm{~J}- \\
& \text { De }[19] \mathrm{J}-, \mathrm{T}_{2-1}=6-(-1)(-1,-1) \quad \mathrm{T}_{2-2}=2 \mathrm{~T}_{2-2} \\
& \mathrm{~J}-,\left(\mathrm{J}-\mathrm{J}_{\mathrm{z}}+\mathrm{J}_{\mathrm{z}} \mathrm{J}-\right)=\mathrm{J}-\left(\mathrm{J}-\mathrm{J}_{\mathrm{z}}+\mathrm{J}_{\mathrm{z}} \mathrm{J}-\right)-\left(\mathrm{J}-\mathrm{J}_{\mathrm{z}}+\mathrm{J}_{\mathrm{z}} \mathrm{J}-\right) \mathrm{J}-= \\
& =J-\left(J-J_{z}\right)-\left(J_{z} J-\right) J-=J-J z J-+J--J-J z-J-J-=2 J-2 \\
& \therefore \mathrm{T}_{2-2}=\mathrm{J}^{2}
\end{aligned}
$$

Para os cálculos acima, usamos as seguintes relações de comutação:

$$
\begin{array}{ll}
\mathrm{J}_{\mathrm{z}}, \mathrm{J}+=\mathrm{J}+ & \mathrm{J}-\mathrm{J}+=\mathrm{J}^{2}-\mathrm{J}_{\mathrm{z}}\left(\mathrm{J}_{\mathrm{z}}+1\right) \\
\mathrm{J}_{\mathrm{z}}, \mathrm{J}-=-\mathrm{J}- & \mathrm{J}+\mathrm{J}-=\mathrm{J}^{2}-\mathrm{J}_{\mathrm{z}}\left(\mathrm{J}_{\mathrm{z}}-1\right) \\
\mathrm{J}+, \mathrm{J}-=2 \mathrm{~J}_{\mathrm{z}} &
\end{array}
$$

Podemos agora calcular os $T_{2 M}(\vec{r})$ por analogia $\operatorname{com}$ os $T_{2 M}(\vec{J})$. Fazendo a correspondência:

$$
\begin{aligned}
& \mathrm{J}_{\mathrm{x}} \longrightarrow \mathrm{x} \quad \mathrm{J}_{+}=\mathrm{J}_{\mathrm{x}}+\mathrm{i} \mathrm{J}_{y} \longrightarrow \mathrm{x}+\mathrm{iy} \\
& \mathrm{J}_{\mathrm{y}} \longrightarrow \mathrm{y} \\
& \mathrm{J}_{z} \longrightarrow \mathrm{z}
\end{aligned}
$$

e verificando relações de comutação como: $J_{x, y}=i z ; J_{x}, y x=i z x$;

$$
\begin{aligned}
& \mathrm{J}_{\mathrm{x}}, \mathrm{x}^{2}=0 \text {, etc... } \\
& \text { podemos escrever: } \mathrm{T}_{22}(\vec{r})=(x+i y)^{2} \\
& \quad \text { Então de [19] } \\
& \mathrm{J}-\mathrm{T}_{22}=2 \mathrm{~T}_{21} ; \mathrm{J}-,(x+i y)^{2}=\left(\mathrm{J}_{x}, i J_{y}\right)(x+i y)^{2}-(x+i y)^{2}\left(J_{x-i} \mathrm{~J}_{y}\right)= \\
& =J_{x}, x^{2}+2 i J_{x}, y x-J_{n}, y^{2}-i J_{y}, x^{2}+2 J_{y}, y x+i J_{y}, y^{2}= \\
& =-2 z x-2 i z y-2 z x-2 i z y=-4 z(x+i y) \\
& \quad \log 0 T_{21}=-2 z(x+i y)
\end{aligned}
$$

Analogamente, calculamos $\mathrm{T}_{20}, \mathrm{~T}_{2-1}, \mathrm{~T}_{2-2}$ e construimos a Tabela I. 
TABELA I. TENSORES IRREDUTIVEIS.

\begin{tabular}{ccc}
\hline$T_{i j}$ & \multicolumn{1}{c}{$T_{2 M}(\vec{J})$} & \multicolumn{1}{c}{$T_{2 M}(\vec{r})$} \\
\hline$T_{22}$ & $J^{2}$ & $(x+i y)^{2}$ \\
$T_{21}$ & $-\left(J z J++J+J_{z}\right.$ & $-2 z(x+i y)$ \\
$T_{20}$ & $\sqrt{2 / 3}$ & $\sqrt{2 / 3}\left(3 z^{2}-r^{2}\right)$ \\
$T_{2-1}$ & $J_{z} J-j-J z$ & $2 z(x-i y)$ \\
$T_{2-2}$ & $J_{-}^{2}$ & $(x-i y)^{2}$ \\
\hline
\end{tabular}

Podemos então resumir a discussão acima, observando que, se uma função de $J_{x}, J_{y}, J_{z} \bar{e}$ um $T_{L M}$, o mesmo serā verdade para uma função de $x, y, z$, desde que sejam verificadas relações de comutação entre as componentes do tipo citadas acima. Deve-se tomar cuidado, entretanto com os possĩveis problemas de comutação entre as componen tes (veja, por exemplo, $T_{21}$ nas duas representações, na Tabela I).

Finalmente, consideremos a aplicação do teorema de Wigner Eckart para elementos de $T_{L M}$ em duas representações quaisquer $p$ e q:

$$
\begin{aligned}
& \left\langle\tau J M_{J}\left|T_{L M}(p)\right| \tau^{\prime} J^{\prime} M_{J}{ }^{\prime}\right\rangle=C\left(J J^{\prime} L J ; M_{J}, M_{J}\right)\left\langle\tau J\left\|T_{L}(\vec{p})\right\| \tau^{\prime} J^{\prime}\right\rangle \\
& \left\langle\tau J M_{J}\left|T_{L M}(q)\right| \tau^{\prime} J^{\prime} M_{J}\right\rangle=C\left(J J^{\prime} L J ; M_{J}, M_{J}\right)\left\langle\tau J\left\|T_{L}(q)\right\| \tau^{\prime} J^{\prime}\right\rangle
\end{aligned}
$$

Comparando as duas expressẽos, vemos que os coeficientes de C.G. são os mesmos. Assim:

$$
\left.\left\langle\tau M_{J} T_{L M}(q) \mid \tau^{\prime} J^{\prime} M_{J}\right\rangle^{\prime}\right\rangle=\left\langle\tau J M_{J}\left|T_{L M}(p)\right| \tau^{\prime} J^{\prime} M_{J} \jmath^{\prime}\right\rangle \frac{\left\langle\tau J|| T_{L}(p) \| \tau^{\prime} J^{\prime}\right\rangle}{\left\langle\tau J|| T_{L}(q)|| \tau^{\prime} J^{\prime}\right\rangle}
$$

CALCULO DOS ELEMENTOS DE MATRIZ DE $H_{Q}$

$$
\begin{array}{r}
\hat{H}_{Q} \bar{e} \text { dado pela expressão } 16: \\
\hat{H}_{Q}=\frac{1}{6} \sum_{i j} Q_{i j}(\text { op }) \frac{\partial E_{j}}{\partial x i}
\end{array}
$$

Temos, então, de calcular os elementos de $Q_{i j}$ (op) na re presentação onde os vetores base são do tipo $\left|I_{m} \tau\right\rangle,\left|I_{m}{ }^{\prime} \tau\right\rangle$.

As componentes do momento angular total do nūcleo são $I_{x}$, $I_{y}, I_{z}$, onde

$$
I_{x}=\sum_{k} l_{x k}+{ }^{2} x k+S_{x k} \text { e analogamente para } I_{y}, I_{z} .
$$


onde ${ }^{2} \times k=$ componente $x$ do momento angular orbital do prōton $k$ e $S_{x k}=$ componente $x$ do momento angular do próton $k$.

As seguintes relações de comutação podem ser verificadas:

$$
{ }^{2} x k, y k=i z k ; \quad s_{x k}, y_{k}=0 \text { etc... }
$$

e darão origem a relações de comutação do tipo:

$$
\begin{aligned}
& I_{x}, y k=i z_{k} \text {, etc... } \\
& \text { Observemos ainda que os termos de } Q_{i j}(o p)=
\end{aligned}
$$

$=e \sum_{k}\left(3 x_{i k} x_{j k}-\delta_{i j} r_{k}^{2}\right)$ são combinações lineares dos $T_{2 M}(\vec{r})$ (ver Tabela I). Alēm disso, a expressão 26 se aplica tambēm a funções que são combinações lineares de $T_{L M}$ de mesmo L.

Assim, considerando as funções

$$
\begin{aligned}
& F(p)=\sum_{M} a_{M} T_{L M}(p) \\
& e \\
& G(q)=\sum_{M} a_{M} T_{L M}(q)
\end{aligned}
$$

teremos

$$
\left\langle\tau J M_{J}|G(q)| \tau^{\prime} J^{\prime} M_{J^{\prime}}\right\rangle=\left\langle\tau J M_{J}|F(p)| \tau^{\prime} J^{\prime} M_{J}{ }^{\prime}\right\rangle \frac{\left\langle\tau J|| T_{L}(q) \| \tau^{\prime} J^{\prime}\right\rangle}{\left\langle\tau J|| T_{L}(p)|| \tau^{\prime} J J^{\prime}\right\rangle}
$$

Fazendo então a correspondência $x i \rightarrow I_{i}$ e $r \rightarrow I$, podemos escrever:

$$
\begin{aligned}
& \left\langle I I \tau\left|e \sum_{k}\left(3 z_{k}^{2}-r_{k}^{2}\right)(I I \tau\rangle=C<\tau I I\right| 3 I_{z}^{2}-I^{2} \mid \tau I I\right\rangle= \\
& =C 3 I^{2}-I(I+1)=C I(2 I-1)[30]
\end{aligned}
$$

onde $C$ é uma constante. C pode ser expressa em termos de um elemen to de matriz, para o qual $m=m^{\prime}=I$ e $i=j=z$ :

$$
\begin{aligned}
& \left.\left\langle\tau I I\left|e \underset{k}{\sum_{k}}\left(3 z_{k}^{2}-r_{k}^{2}\right)\right| \tau I I\right\rangle=C<\tau I I\left|3_{z}^{2}-I^{2}\right| \tau I I\right\rangle=C 3 I^{2}-I(I+1)= \\
& =C I(2 I-1)
\end{aligned}
$$

Podemos agora definir o símbolo eQ $=\langle\tau$ II $|$ e $\underset{k}{\sum_{k}\left(3 z_{k}^{2}-r_{k}^{2}\right)|\tau I I\rangle}$

e teremos

$$
C=\frac{e Q}{I(2 I-1)}
$$

Como estamos tratando aqui de elementos de matriz internos 
a um conjunto de números quânticos $\tau$ e $I$, podemos usar os resultados $[30]$ e $[32]$ para substituir $Q_{i j}$ (op) na Hamiltoniana:

$$
H_{Q}=\frac{e Q}{6 I(2 I-1)} \sum_{i j} \frac{\partial E}{\partial x i} \frac{3}{2}\left(I_{i} I_{j}+I_{j} I_{i}\right)-\delta_{i j} I^{2}
$$

A interação quadrupolar descrita acima aplica-se a uma orientação arbitraria dos eixos coordenados. Entretanto 0 tensor $\frac{\partial E_{j}}{\partial x_{j}} \equiv v_{j i}$ pode ser simplificado pela escolha de um conjunto de e $\underline{i}$ xos tal que $v_{j i}=0$ se $i \neq j$. Teremos:

$$
H_{Q}=\frac{e Q}{6 I(2 I-1)} V_{x x}\left(3 I_{x}^{2}-I^{2}\right)+V_{y y}\left(3 I_{y}^{2}-I^{2}\right)+V_{z z}\left(3 I_{2}^{2}-I^{2}\right)
$$

se usarmos a equação de Laplace $\underset{j}{\sum_{i j}} V_{i j}=0$, esta relação pode ser rees crita:

$$
H_{Q}=\frac{e Q}{4 I(2 I-1)} V_{z z}\left(3 I_{z}^{2}-I^{2}\right)+\left(V_{x x}-V_{y y}\right)\left(I_{x}^{2}-I_{y}^{2}\right)
$$

De fato, de 34 , usando a equação de Laplace, virā:

$$
\begin{aligned}
& \frac{1}{6} V_{x x}\left(3 I_{x}^{2}-I^{2}\right)+V_{y y}\left(3 I_{y}^{2}-I^{2}\right)+V_{z z}\left(3 I_{z}^{2}-I^{2}\right)=\frac{1}{2}\left(V_{x x} I_{x}^{2}+V_{y y} I_{y}^{2}+\right. \\
& \left.+V_{z z} I_{z}^{2}\right)=\frac{1}{4} V_{x x^{2}}^{2} I_{x}^{2}+2 V_{y y} I_{y}^{2}+V_{z z}\left(3 I_{z}^{2}-I^{2}\right)=
\end{aligned}
$$

obs: $\quad 2 I_{z}^{2}=\left(3 I_{z}^{2}-I^{2}\right)+I_{x}^{2}+I_{y}^{2}$

$$
\begin{aligned}
\therefore \quad & \frac{1}{4} V_{x x} 2 I_{x}^{2}+V_{y y} 2 I_{y}^{2}+V_{z z}\left(3 I_{z}^{2}-I^{2}\right)-\left(V_{x x}+V_{y y}\right)\left(I_{x}^{2}+I_{y}^{2}\right)= \\
& =\frac{1}{4} V_{z z}\left(3 I_{z}^{2}-I^{2}\right)+V_{x x} I_{x}^{2}+V_{y y} I_{y}^{2}-V_{x x} I_{y}^{2}-V_{y y} I_{x}^{2}= \\
& =\frac{1}{4} V_{z z}\left(3 I_{z}^{2}-I^{2}\right)+\left(V_{x x}-V_{y y}\right)\left(I_{x}^{2}-I_{y}^{2}\right)
\end{aligned}
$$

Assim, vemos de [35] que apenas 2 parâmetros são necessāa rios para caracterizar as derivadas do potencial: $v_{z z} e\left(v_{x x}-v_{y y}\right)$. E conveniente definir agora 2 parâmetros:

parâmetros de assimetria

$$
n=\frac{v_{x x}-v_{y y}}{v_{z z}}
$$

gradiente de campo eq $=V_{z z}$

Se a simetria for axial, teremos $n=0$ pois então $v_{x x}=v_{y y}$. 
CAMPO FORTE: EXEMPLO

Suponhamos que um campo magnético seja aplicado sobre um átomo, e que tal campo tenha simetria axial (ou outra simetria tal que $\left.v_{x x}=v_{y y}\right)$. Suponhamos ainda que o campo seja aplicado segundo uma direção $Z^{\prime}$, e que o núcleo seja orientado segundo $Z$.

A Hamiltoniana para a interação do momento magnētico do núcleo com o campo aplicado Ho, e interação quadrupolar é:

$$
H=\gamma n h H o I z^{\prime}+\frac{e^{2} g Q}{4 I(2 I-T)}\left(3 I_{z}^{2}-I^{2}\right)
$$

onde $\gamma$ n è o raio giromagnētico do nūcleo.

Trataremos aqui do caso em que a interação magnētica ē for te em relação à interação quadrupolar. Faremos aqui o tratamento da interação quadrupolar como perturbação. Vamos escolher $x$ e $x^{\prime}$ no mes mo plano de $z$ e $z$ '; então, podemos ver da Figura 2 que

$$
I_{z}=I_{z}, \cos \theta+I_{x^{\prime}} \operatorname{sen} \theta
$$

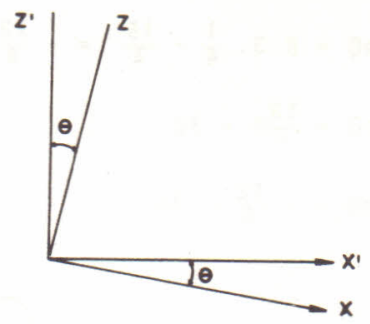

$[39]$

Figura 2. Campo aplicado segundo uma direção $z^{\prime}$, com nūcleo orienta do segundo $z$.

Levando 38 em 39 , teremos

$H=-\gamma n h H o I z^{\prime}+\frac{e^{2} q Q}{4 I(2 I-1)} \quad 3 I_{z^{2}}^{2} \cdot \cos ^{2} \theta+3 I_{x}^{2} \cdot \operatorname{sen}^{2} \theta+$

$+\left(I_{z^{\prime}} I_{x^{\prime}}+I_{z^{\prime}} I_{x^{\prime}}\right) 3 \operatorname{sen} \theta \cos \theta-I^{2}$

Em primeira ordem, I $z^{\prime}$, é diagonal; I $x^{\prime}$ tem elementos dia gonal nulos: então termos do tipo $I_{z^{\prime}}, I_{x^{\prime}}$, em primeira ordem, não contribuem. Mas, $I_{X^{\prime}}^{2}$, tem elementos da diagonal não nulos. Alēm di $\underline{s}$ so, expressando $I_{x^{\prime}}{ }^{\prime}=\frac{1}{2}\left(I_{+}^{\prime}+I_{-}^{\prime}\right)$ e $I_{y^{\prime}}=\frac{1}{2 i}\left(I_{+}^{\prime}-I_{-}\right)$pode-se mos trar que os elementos da diagonal de $I_{x}^{2}$, e $I_{y}^{2}$, são iguais:

$$
\left\langle m\left|I_{x}^{2}\right| m\right\rangle=\left\langle m\left|I_{y}^{2}\right| m\right\rangle=\frac{1}{2}\left\langle m\left|I_{-}^{2} I_{z}^{2},\right| m\right\rangle=\frac{1}{2} \quad I(I+1)-m^{2} \quad[4]
$$

Então

$$
<m|H| m>=E m=-\gamma n h H o m+\frac{e^{2} q Q}{4 I(2 I-1)}\left(\frac{3 \cos ^{2} \theta-1}{2}\right) 3 m^{2}-I(I+1)
$$


Aplicąão para um átomo ou ion de estado fundamental ${ }^{2} \mathrm{~S}_{1 / 2}$ e spin nuclear $3 / 2$ num campo magnético forte

Neste caso jā conhecemos o cālculo de abertura de nīveis, incluindo o termo -rnhHom dado em [42] (3).

Resta então apenas calcular o desvio nos níveis, causado pela interação quadrupolar:

$$
E m Q=\frac{e^{2} q Q}{4 I(2 I-1)}\left(\frac{3 \cos ^{2} \theta-1}{2}\right) 3 m^{2}-I(I+1)
$$

Para simplificar, façamos $\beta=\frac{e^{2} q Q}{12}\left(\frac{3 \cos ^{2} \theta^{-1}}{2}\right)$

$$
\therefore E m Q=\beta 3 m^{2}-I(I+1)=\beta 3 m^{2}-\frac{15}{4}
$$

$$
\begin{array}{ll}
\text { Para } m=3 / 2 & E m Q=\beta 3 \cdot \frac{9}{4}-\frac{15}{4}=\frac{12}{4} \beta=3 \beta \\
\text { Para } m=1 / 2 \quad E m Q=\beta 3 \cdot \frac{1}{4}-\frac{15}{4}=-\frac{12}{4} \beta=-3 \beta \\
\text { Para } m=-3 / 2 \quad E m Q=\frac{12}{4} \beta=3 \beta \\
\text { Para } m=-1 / 2 \quad E m Q=-\frac{12}{4}=-3 \beta
\end{array}
$$

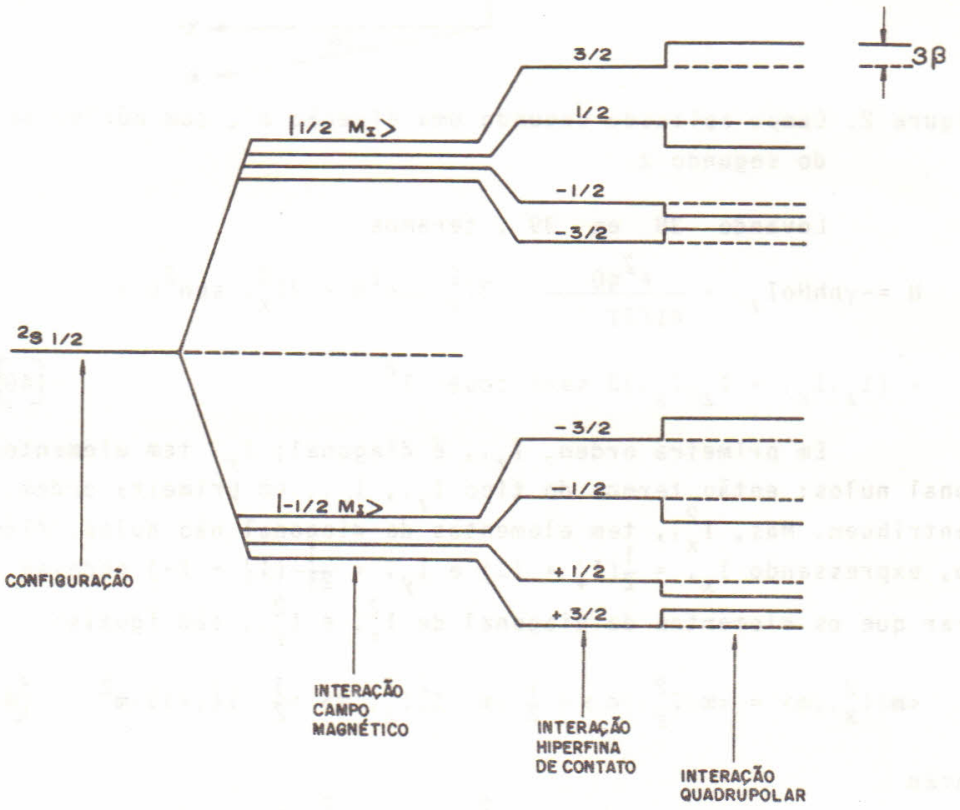

Figura 3. Efeito da ação quadrupolar sobre a estrutura fina de contato. 


$$
H=-\gamma n h \mathrm{Hom}_{2}+\frac{\mathrm{e}^{2} \mathrm{qQ}}{41(2 \mathrm{I}-1)}\left(3 \mathrm{~m}_{2}^{2}-\mathrm{I}^{2}\right)
$$

A interação campo magnētico è muito maior que a interação de quadrupolo, que será então estudada como perturbação da anterior, em primeira ordem.

os nĩveis se abrirão devido à interação campo magnētico da da por -үnhHom ro $I=3 / 2$ e $I=1 / 2$, dadas por:
$I=3 / 2$
$|1 / 2,+1 / 2\rangle$
$I=3 / 2$
$|3 / 2,3 / 2\rangle$
$|3 / 2,1 / 2\rangle$
$|1 / 2,-1 / 2\rangle$
$|3 / 2,-1 / 2\rangle$
$|3 / 2,-3 / 2\rangle$

As energias dos níveis serão dadas por -rnhHom e a inte ração de quadrupolo apenas deslocará os níveis.

$$
\begin{aligned}
& \text { Teremos: } \quad I=3 / 2: m_{I}=3 / 2 \longrightarrow-3 / 2 \gamma n h H o \\
& m_{I}=1 / 2 \longrightarrow-1 / 2 \gamma n h H o \\
& m_{I}=-1 / 2 \rightarrow+\frac{1}{2} \gamma \mathrm{nhHO} \\
& m_{I}=-3 / 2 \rightarrow \frac{3}{2} \gamma n h H o \\
& I=1 / 2 \quad m_{I}=1 / 2 \Longrightarrow-\frac{1}{2} \mathrm{\gamma nhHo} \\
& \mathrm{m}_{\mathrm{I}}=-1 / 2 \Longrightarrow \frac{1}{2} \mathrm{\gamma nhHo} \\
& \text { e os deslocamentos quadrupolares, } \frac{e^{2} q Q}{4 I(2 I-T)} 3 m_{I}^{2}-I(I+1) \\
& \begin{aligned}
I=3 / 2, m_{I} & =3 / 2 \Longrightarrow 3 / 8 e^{2} q Q \\
m_{I} & =1 / 2 \Longrightarrow 1 / 8 e^{2} q Q \\
m_{I} & =-1 / 2 \Longrightarrow 1 / 8 e^{2} q Q \\
m_{I} & =-3 / 2 \Longrightarrow 3 / 8 e^{2} q Q
\end{aligned}
\end{aligned}
$$

A Figura 6(a) mostra o esquema de niveis obtido experimen talmente. Observe que os deslocamentos quadrupolares têm sinal con trārio aos calculados aqui. Isto permite concluir que e ${ }^{2} q Q$ tem sí nal negativo. A Figura 6(b) mostra o espectro Myssbauer correspon dente, jā levando-se em consideração a interação quadrupolar. As lị nhas pontilhadas indicam a posição dos picos na ausēncia da intera ção quadrupolar. Os desvios são calculados aqui, analogicamente ao exemplo citado.

Conhecidas as energias de cada $m_{I}$ da interação hiperfina, poderemos obter, através do valor de $\Delta E$ medido no espectro, o valor do produto $q Q$, onde 
Interaçõo quadrupolar no Efeito MBssbauer* : Exemplo

Examinaremos primeiro o caso do Biferrocenil, onde não há campo magnético interno.

Temos o estado fundamental $I=1 / 2$ e 0 estado excitado $I=3 / 2$. 0 esquema de níveis será (Figura 4 ).

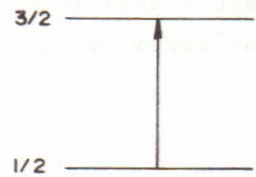

(a) e o espectro Mössbauer correspondente seró

(b)

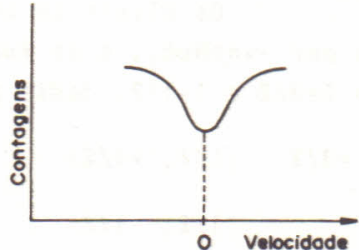

Figura 4. Esquema de níveis para $I=1 / 2$ e $I=3 / 2$.

Consideremos agora a interação quadrupolar (Figura 5).

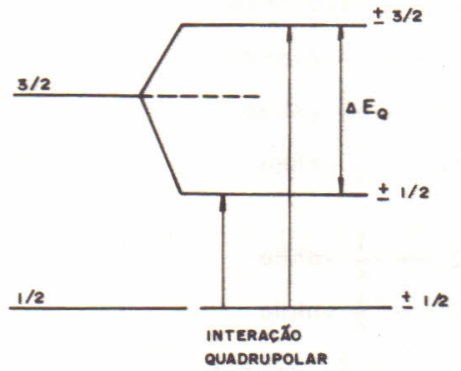

(a)

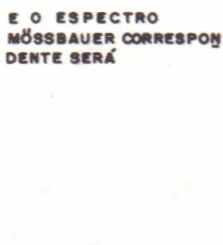

MUSSBAUER CORRESPON MOSSBAUER

Figura 5. Esquema de nîveis com (a) interação quadrupolar; (b) Efei to Mussbauer correspondente.

Os autovalores da Hamiltoniana serão:

$E Q\left(M_{I}\right)=e^{2} q Q \frac{3 M_{I}^{2}-I(I+1)}{4 I(2 I-I)}$

e $\Delta E_{Q}=E_{Q}(3 / 2)-E_{Q}(1 / 2)=\frac{e^{2} q Q}{2}$

observamos, então que a abertura do pico na Figura 4 nos dois picos da Figura 5 dá uma medida da interação quadrupolar.

Podemos examinar agora 0 caso do $\mathrm{Fe}_{2} \mathrm{O}_{3}$, onde teremos uma interação magnética hiperfina (Efeito Zeemann).

A Hamiltoniana do problema é dada por 38 , na aproxima ção campo forte.

* Não levaremos em consideração nos espectros e diagramas o "desvio isomérico", quase sempre presente nos espectros Mossbauer. 
$q=\frac{1}{e} V_{z z}=\frac{1}{e} \frac{\partial^{2} v}{\partial z^{2}}$. Assim, se desejamos calcular o momento de quadrupolo do núcleo é necessārio conhecer o valor do gradiente de campo, e vice-versa. Entretanto, o que interessa ao Efeito Mossbauer é o valor de e $(=\triangle E Q)$ que dará informações qualitativas sobre a vị zinhança eletrōnica do nūcleo e o tipo de rede do sōlido.

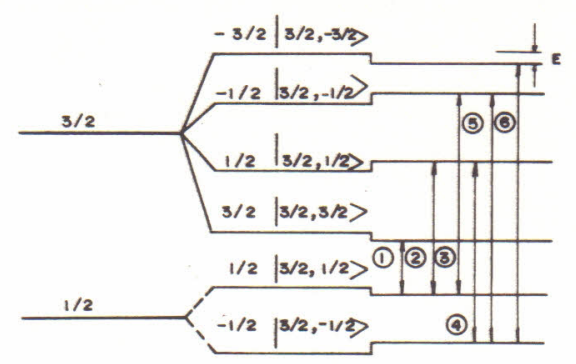

(a)

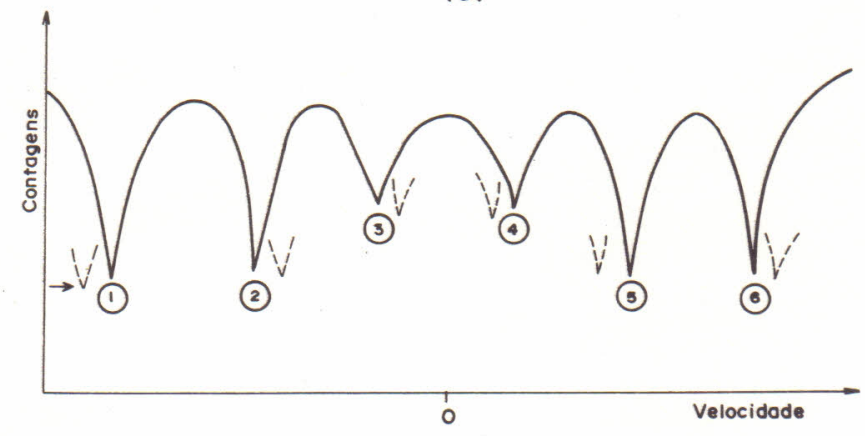

(b)

Figura 6. (a) Esquema de níveis obtidos experimentalmente, com os deslocamentos quadrupolares correspondentes.

(b) Espectro Mossbauer correspondente ao Esquema (a) leva da em conta a interação quadrupolar.

CONCLUSÃO

Do exposto vemos que os níveis por interações quadrupola res podem ser detectados experimentalmente pela espectroscopia Mossbauer, o qual mostra as diferenças $\Delta E_{Q}$, de energia entre os níveis m nuclea res, deslocados pela interação quadrupolar.

A tēcnica da espectroscopia Mossbauer determina experimen talmente as diferenças de energia entre estes deslocamentos.

BI BL I OGRAF I A

1. MESSIAH,A. Quantum Mechanies. Tomo 1 e 2. Dunod Editeurs, Paris. 1945. 
2. JACKSON,J.D. Classical Eletrodynamics. Second Edition. John Wiley and Sons, Inc., New York, 1975.

3. BAYM,G. Lectures on Quantum Mechanies. W.A. Benjamin, Inc., New York. 1969.

Recebido em agosto, 1982; aceito em setembro, 1982. 